\title{
UNA INSCRIPCIÓN FUNERARIA DE INIESTA (CUENCA) Y DOS PONDERA CON INSCRIPCIÓN DEL MuSEo Municipal DE REQuENA
}

ASUNCIÓN MARTÍNEZ VALLE 우

Museo Municipal de Requena.museomunicipal@requena.es

\section{INSCRIPCIÓN FUNERARIA DE INIESTA (CUENCA)}

\section{CIRCUNSTANCIAS DEL HALLAZGO}

La inscripción que presentamos procede del término municipal de Iniesta pero se conserva en Campillo de Altobuey. Se recuperó hace aproximadamente unos 25 años, tras unas labores agrícolas, en una de las propiedades de D. Gregorio Carrión, abogado de Iniesta. Cuando se encontró la inscripción ya había sido vaciada por su parte posterior para utilizarla como pila para recoger agua por lo que se supone ya estaba fuera de su contexto original.

El párroco de Campillo de Altobuey, amigo del propietario, coleccionaba pilas de piedra, muy características de la labor tradicional de cantería en esta población, así que D. Gregorio la trasladó a Campillo y se la regaló. En una reunión de amigos en que se encontraban D. Gregorio, el párroco y Santiago Montoya, historiador del arte, se comentó que una de las pilas tenía letras. Santiago Montoya pidió verla y comprendió que se trataba de una lápida funeraria romana. La sacaron del almacén, la colocaron sobre un pedestal y la pusieron en el jardín de la vivienda familiar como un motivo ornamental.

En primavera de 2015 nos desplazamos a Campillo con Juan Manuel Abascal, a quien agradecemos su colaboración en este trabajo, para documentar la inscripción. Esta se encontraba en la casa familiar, ya deshabitada, cubierta de líquenes y musgos como consecuencia de estar los últimos años a la intemperie y en una zona de umbría. Comentamos la necesidad de trasladarla a un emplazamiento cubierto y hemos podido verificar que en la actualidad se encuentra en el Museo de Campillo, junto con otras inscripciones recuperadas en la zona (Martínez Valle 1999: 437-440).

\section{DESCRIPCIÓN}

La inscripción está realizada sobre un bloque de caliza de Buixcarró, que mide 80 x 51 x $42 \mathrm{~cm}$. En su cara frontal presenta el texto en dos líneas y un remate circular en el tercio superior que rebaja la mitad del espesor del bloque y da la apariencia, desde su parte frontal, de ser una estela. La parte posterior del bloque conserva sus dimensiones originales, pero está parcialmente vaciada para poder utilizarla como pila de uso doméstico. 
El texto dice lo siguiente:

\section{C(aius) Roscius}

C(aii) L(ibertus) Lucretius

Traducción: Cayo Roscio Lucrecio, liberto de Cayo

Las letras son capitales cuadradas de $6 \mathrm{~cm}$ de altura, tienen ápices que se aprecian mejor en la letra $\mathrm{V}$ y presenta un nexo de ET en la segunda línea.

\section{COMENTARIO}

Se trata de un liberto de la gens Roscia, del que toma el praenomen, Caius, y el nomen. La gens Roscia está documentada en Hispania en pocos casos y se concentra en el SO peninsular, en Córdoba, Cáceres y con más frecuencia en Mérida (Abascal 1994: 210-211). El cognomen de Caius Roscius también es un nomen Lucretius. En este caso es un gentilicio más frecuente (Abascal 1994: 175176) que está muy extendido en Hispania, pero solamente en otro caso, que conozcamos, aparece en posición de cognomen: una mujer documentada en Barcelona ( $A E$ 1966: 200).

Que aparezcan dos gentilicios en los tria nomina del difunto pensamos que podría ser indicativo de una relación estrecha o de parentesco entre ambas familias. Aparte de esta hipótesis poco más podemos decir de esta inscripción que no presenta fórmulas funerarias ni dedicación a los dioses Manes, simplemente el nombre del difunto y su condición social. Sobre la cronología podemos señalar que el nombre del difunto va en nominativo, la simplicidad del texto y los caracteres epigráficos lo que nos permite datar el momento de su labra de mediados del s. I d.C.

Iniesta (Cuenca) se localiza en una vía natural de comunicación entre las ciudades romanas de la costa valenciana y las del interior de la provincia de Cuenca, como son Valeria y Segobriga (Palomero 1987). Esta población se identifica con la ceca ibérica de $\operatorname{IKALE}(N) S K E N$ (Martínez Valle 1994: 59-66; Ripollès 1997: 145-168) y posterior Segestica (Almagro Basch 1984: 31). El descubrimiento de restos romanos en Iniesta confirma la ocupación durante este periodo. En este sentido tenemos que citar las tres inscripciones funerarias conocidas hasta la actualidad (Colmenero 1982: 230; Valero 1995) y esta última que presentamos del entorno de la ciudad.

\section{DOS PONDERA DEL MUSEO MUNICIPAL DE REQUENA}

Los pondera que presentamos proceden de los fondos antiguos del museo. Ambas piezas se recuperaron revisando los almacenes y no presentaban siglado, por lo que son de procedencia incierta. Consultamos el primer catálogo del museo (Aparicio y Latorre 1977) y un pequeño inventario manuscrito de 1988 que firma María Vicenta Sanmartín Nácher y solo encontramos referencias a dos grupos de pondera de dos yacimientos romanos que se prospectaron en los años en que se fundó el museo.

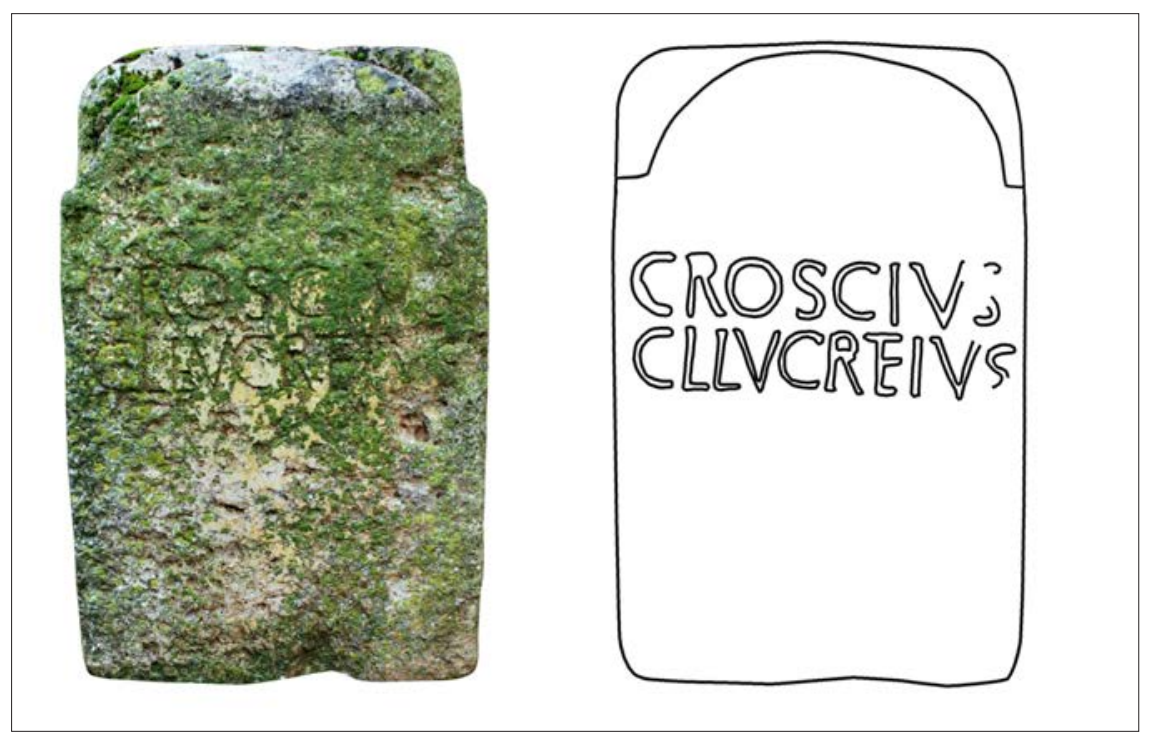

Fig. 1: Fotografía y dibujo de la inscripción de Iniesta. 
A pesar de que no existe ningún tipo de identificación, este inventario cita la existencia de pondera romanos en La Pajarilla, en la vega del Magro, y en El Rebollar, suponemos de la villa romana de Las Paredillas.

Los pondera son instrumentos relacionados con los telares y se utilizaban para tensar la urdimbre sobre la que se tejía la trama que va conformando el tejido. El primero de los pondera tiene forma tronco-piramidal, varias fracturas en su parte posterior y pesa $295 \mathrm{~g}$. En el tercio superior, en su parte lateral, presenta la característica perforación que atraviesa la pieza por donde se sujetaría a los hilos de la urdimbre, que en este caso está enmarcada por un círculo que muestra huellas de uso y mucho desgaste.

Destaca por su inscripción en letra cursiva realizada antes de su cocción. Tanto el desgaste del pondus como las fracturas que presenta no impiden la lectura, si bien podría existir una última letra fracturada.

La primera letra se ve claramente que es una $\mathrm{S}$ con un tamaño superior a las otras. Le sigue una $\mathrm{E}$ que son dos líneas verticales paralelas que no presenta problemas de interpretación, una V, y otra E con la misma grafía; así que se identifica fácilmente la primera parte del nombre SEVE. El resto de la inscripción presenta más dificultades de lectura por encontrarse las letras menos marcadas y por el desgaste del lateral derecho que podría haber eliminado parte de un último signo. La quinta letra podría ser una R, ligeramente desplazada y también de un tamaño más grande; la sexta presenta más dudas, podría ser una A y junto a ella una línea vertical que podría pertenecer a una $\mathrm{E}$ incompleta. En ese caso se podría leer Severa $[e]$; no obstante cabría la posibilidad de que se hubiese omitido la A, ya que no se pronuncia y es común no escribirla en las inscripciones en cursiva, y que los signos finales fueran una $\mathrm{E}$ (dos líneas verticales). La lectura que proponemos es:

\section{Sever $(a) e$}

El cognomen Severus/a es frecuente en Hispania, con una distribución muy regular (Abascal 1994: 506-507). En este caso es la primera vez que se documenta en la Meseta de Requena-Utiel. Que aparezca en genitivo sería indicativo de pertenencia a una mujer.

El otro pondus también tiene forma tronco-piramidal y pesa 310 g. En su tercio superior presenta también la perforación que caracteriza estas piezas y el lateral derecho se aprecia más desgastado por el uso.

En la parte superior se ve una estampilla también ante cocturam muy bien marcada: un círculo inciso con varias

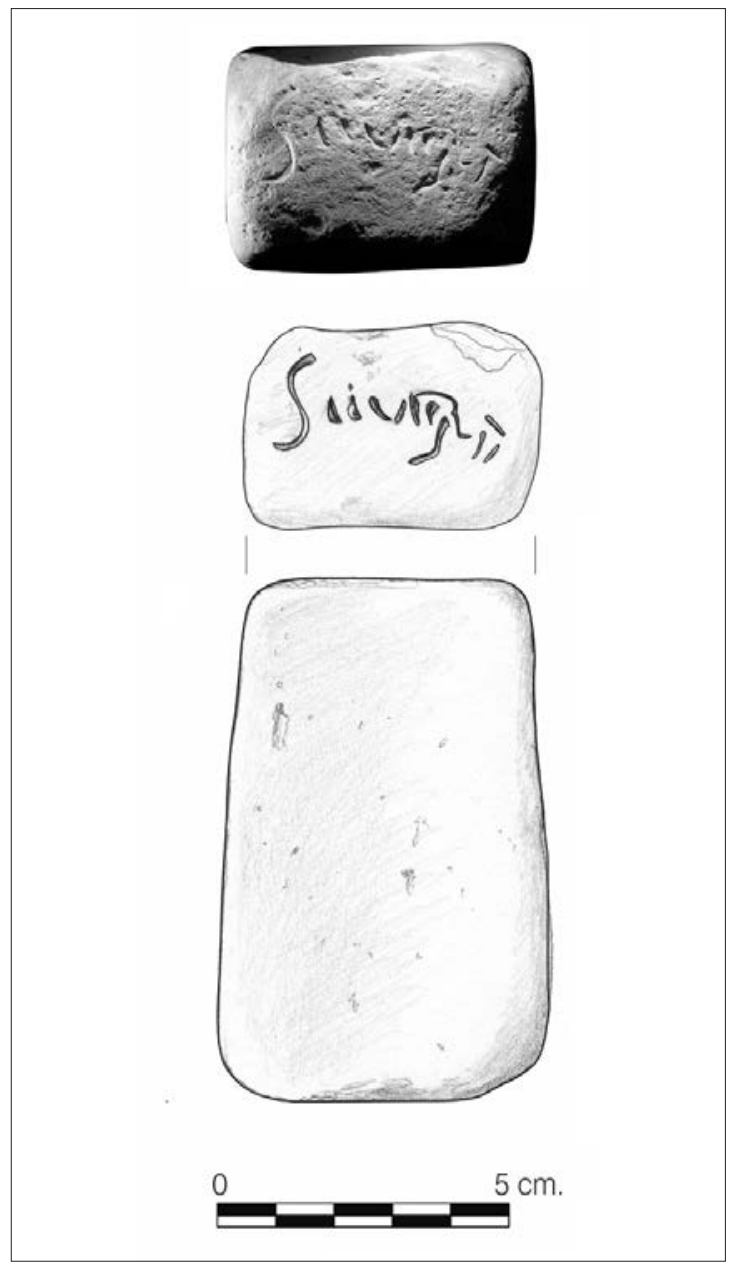

Fig. 2: Fotografía y dibujo del pondus de Severa.

letras de un nombre que no presenta problemas de interpretación, a pesar de que varias fracturas han dañado la estampilla, desapareciendo algunas letras del centro y parte del círculo sobre el que están escritas.

En la estampilla circular se pueden leer una $\mathrm{C}$, una A, una $\mathrm{E}$ y una $\mathrm{C}$; al otro lado de la fractura una $\mathrm{A}$ y una $\mathrm{E}$ con nexo, por lo que el texto podría ser:

\section{Caec(ili)ae}

El espacio fracturado estaría ocupado por las tres letras I, L e I que completarían el nombre de Caecilia en genitivo, indicativo de pertenencia, como en el caso anterior, a otra mujer. Al tratarse un sello aplicable a otros soportes podría identificar un taller o un fundus de los Caecilii. 


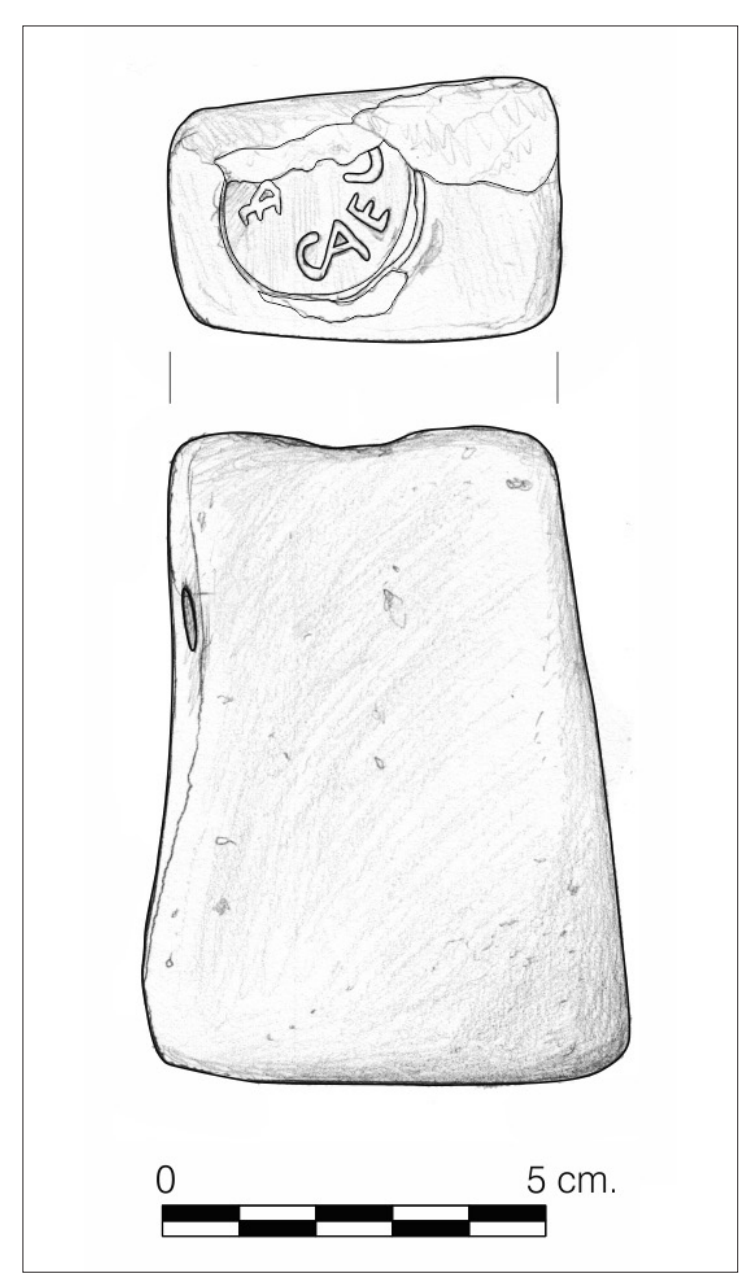

Fig. 3: Dibujo del pondus de Caecilia.

La gens Caecilia está bien atestiguada en Hispania (Abascal, 1994: 98-102). Aunque en el territorio de Valentia no es especialmente frecuente, en el territorio de Edeta los hemos encontrado en 13 ocasiones (Corell 1996: 236). En Requena se documentan en un pedestal reutilizado en la la construcción de la muralla islámica del Patio de Armas (Martínez Valle 1998: 263-265). Se trata de la inscripción funeraria de Caecilia Titis, liberta de Caius Caecilius, dedicada por su hermano Fabius Messenius, donde el texto evidencia su condición de libertos y la relación de parentesco. La inscripción especifica que fueron manumitidos por familias diferentes, donde el dedicante, al igual que en la inscripción de Iniesta, también tiene un nomen en posición de cognomen.
Los Messenii fueron una de las familias nobles de la ciudad de Valentia, presentes en dos inscripciones situadas en el foro de esta ciudad: una de carácter monumental que señala que un miembro de esta familia dedicó un templo a una divinidad que se desconoce al estar incompleta la inscripción, y la otra en un pedestal honorífico (Corell 1997: 101-103).

La relación de las familias, en este caso se puede demostrar a través del soporte de la lápida de Titis, hermana de Messenius, decorada con el mismo motivo que la inscripción honorífica de Marcia Postuma Messenia Lucilla, localizado en el foro de la ciudad de Valentia. En ambos casos un gran rombo ocupa la parte lateral de la inscripción, lo que nos hace pensar la posibilidad de que el dedicante encargase el soporte de la inscripción de su hermana en el mismo taller que Lucilla (Cebrián 2000: 121) y que los Messenii de Valentia, tuviesen una villa en lo que hoy es Requena.

La relación de los Messenii con este territorio está documentada también a través de la inscripción funeraria de Messenia Onesiphoris (Corell 1997: 215-217) recuperada también en esta ciudad. Coincide que ambas mujeres, por sus cognomina, podrían ser esclavas de origen griego (Solin 1982: 547) al igual que podría ser el origen de Fabius Messenius. De todas maneras, con los Flavios y los Antoninos, se extendió la moda de los nombres griegos entre libertos y población servil (Abascal 1994: $35)$ y no se puede garantizar su origen.

Volviendo a los pondera, comentar que tradicionalmente la elaboración de los tejidos se consideraba una labor noble que ensalzaba el trabajo del lanificium por parte de las mujeres (Beltrán Lloris y Beltrán Lloris 2012: 136-137), lo que confirman los dos pondera, uno inciso y otro estampillado, que presentamos en este trabajo (Antolini y Marengo 2012: 152).

\section{BIBLIOGRAFÍA}

ABASCAL, J. M. (1994): Los nombres personales en las inscripciones latinas de Hispania, Murcia.

$A E=L ` A n n e ́ e ~ E p i g r a p h i q u e$.

ALMAGRO BASCH, M. (1984): Segobriga. Inscripciones ibéricas, latinas paganas y latínas cristianas, Excavaciones arqueológicas de España.

ANTOLINI, S.; MORENGO, S. M. (2012): Scrivere i pesi da telaio. La documentazione dell'Italia romana, Sylloge Epigraphica Barcinonensis X,149-168.

APARICIO, J.; LATORRE, F. (1977): Catálogo-Guía del Museo Arqueológico de Requena (Valencia-España), Requena. 
BELTRÁN LLORIS, F.; BELTRÁN LLORIS, M. (2012): Ama lateres! Sobre una pesa de telar cesaraugustana relativa al lanificium, Sylloge Epigraphica Barcinonensis X, $127-184$.

CEBRIÁN, R. (2000): Titulum fecit. La producción epigráfica romana en las tierras valencianas, Madrid.

COLMENERO, A. (1982): Cuenca romana, contribución al estudio epigráfico, Lucentum I, 271-281.

CORELL, J. (1996): Inscripcions romanes de Valentia i el seu territori, València.

CORELL, J. (1997): Inscripcions romanes d'Edeta i el seu territori, València.

MARTÍNEZ VALLE, A. (1994): En torno a la localización de la ceca de Ikalesken, Actas del IX Congreso Nacional de Numismática, Elche, 59-66.
MARTÍNEZ VALLE, A. (1998): Nuevos hallazgos de inscripciones romanas en la provincia de Valencia, Saguntum-PLAV 31, 263-265.

MARTÍNEZ VALLE,A. (1999): Cuatro inscripciones funerarias romanas procedentes de Campillo de Altobuey (Cuenca), Actas del XXV Congreso Nacional de Arqueología, Valencia, 437-440.

PALOMERO, S. (1987): Las vías romanas de la provincia de Cuenca, Cuenca.

RIPOLLÈS ALEGRE, P. P. (1997): De nuevo sobre la localización de Ikale(n)sken, $1^{\text {As }}$ Jornadas de arqueología ibérica de Castilla-La Mancha. Iniesta (Cuenca), Toledo, 145-168.

SOLIN, H. (1982): Die griechischen Personennamen in Rom. Ein Namenbuch, Berlín.

VALERO, M. Á. (1995): Una inscripción funeraria procedente de Iniesta (Cuenca), Saguntum-PLAV 25, 283-286. 